\section{(C) OPEN ACCESS}

\title{
Rare case of atypical crescentic glomerulonephritis and interstitial lung disease with negative anti-GBM antibody and positive anti-MPO antibody
}

\author{
Alexander Hanna, ${ }^{1}$ Jenny Ross, ${ }^{2}$ Fernanda Heitor ${ }^{3}$
}

${ }^{1}$ Medical School, Northwestern University Feinberg School of Medicine, Chicago, Illinois, USA ${ }^{2}$ Department of Pathology, Northwestern Memorial Hospital, Chicago, Illinois, USA ${ }^{3}$ Department of Medicine, Northwestern Memorial Hospital, Chicago, Illinois, USA

\section{Correspondence to Alexander Hanna,} AlexBHanna@gmail.com

Accepted 20 June 2019

\section{Check for updates}

(C) BMJ Publishing Group Limited 2019. Re-use permitted under CC BY-NC. No commercial re-use. See rights and permissions. Published by BMJ.

To cite: Hanna A, Ross J, Heitor F. BMJ Case Rep 2019:12:e229256. doi:10.1136/bcr-2019229256

\section{SUMMARY}

A 70-year-old man presented with 1 month of haematuria and mild right-sided flank pain with no other symptoms. Diagnostic workup included serum studies which showed the presence of antimyeloperoxidase antibodies, a kidney biopsy which demonstrated necrotising crescentic glomerulonephritis with linear immunofluorescence of the basement membrane, and electron microscopy which exhibited thickening of the glomerular basement membrane. Incidentally, the patient was discovered to have a latent hepatitis B infection, which complicated immunosuppressive therapy. He was treated with a course of plasmapheresis and methylprednisolone, followed by entecavir for hepatitis B prophylaxis, and finally by rituximab. This case of glomerulonephritis was notable for its resemblance to the better known Goodpasture's disease. Typically, Goodpasture's syndrome exists on a spectrum from seronegative disease to double-positive disease that presents with both anti-glomerular basement membrane (anti-GBM) and cytoplasmic-antineutrophil cytoplasmic antibodies/antiproteinase 3 antibodies (c-ANCA/antiPR3). However, this patient's glomerulonephritis was unique because he presented negative for anti-GBM antibodies and positive for perinuclear-antineutrophil cytoplasmic antibodies/antimyeloperoxidase antibodies (p-ANCA/anti-MPO).

\section{BACKGROUND}

This case was a true diagnostic mystery, especially since most clinicians are familiar with the already rare condition colloquially known as Goodpasture's disease, but not the more unusual cases of glomerulonephritis such as in this patient. Insights from this case may assist other clinicians in diagnosing unique presentations of glomerulonephritis and formulating treatment plans for patients on immunosuppressive therapy.

\section{CASE PRESENTATION}

A 70-year-old man presented with 1 month of haematuria and mild right-sided flank pain. He described his urine as first appearing 'muddy red' 1 month ago with no change in frequency of voiding or volume of urination from his baseline. He denied seeing any blood clots in the urine, experiencing dysuria, or new onset incontinence. In the emergency department (ED), a Foley catheter was placed, and pink urine was noted in the bag. In addition, he denied all other symptoms during an extensive review of symptoms including haemoptysis, shortness of breath, chest pain and fever.

His medical history is most significant for prostate cancer treated with prostatectomy 4 years ago, followed by recurrence and treatment with 4 months of radiation therapy completed 2 weeks prior to admission. Of note, the radiation therapy fields did not include his kidneys. In addition, he has a history of stage III chronic kidney disease (CKD), hypothyroidism, vitiligo, hypertension, gastro-oesophageal reflux disease and gout.

His social history included daily occupational exposure to radiation in the aerospace industry, immigration from southeast Asia and no use of tobacco, alcohol or recreational drugs.

On exam, the patient generally appeared well. His vital signs were notable for a blood pressure of $180 / 76 \mathrm{~mm} \mathrm{Hg}$, heart rate of 68 beats/min, respiratory rate of 18 breaths/min, temperature of $96.9^{\circ} \mathrm{F}\left(36.1^{\circ} \mathrm{C}\right)$ and oxygen saturation of $97 \%$ on room air. The only abnormal finding on his physical exam was general skin pallor as well as patches of hypopigmentation throughout the body. Of note, there was no suprapubic tenderness, costovertebral angle tenderness or trauma at the urethral meatus.

\section{DIFFERENTIAL DIAGNOSIS}

The differential diagnosis for gross haematuria is broad and begins with a urinalysis (UA) to confirm the presence of red blood cells (RBCs) and rule out mimickers such as myoglobin from rhabdomyolysis. In this case, the UA showed 11-50 RBCs per high-powered field, suggesting that blood, not myoglobin, was being lost in the urine.

In addition, a complete blood count was obtained and showed an anaemia with haemoglobin ( $\mathrm{Hgb})$ of $67 \mathrm{~g} / \mathrm{L}$ and haematocrit of $20.3 \%$ (table 1 ). The mean corpuscular volume (MCV) was slightly elevated at $102 \mathrm{FL}$; however, the patient's baseline MCV was high in the 90s, and repeat testing was within the normal limits. As a precaution, causes of macrocytic anaemia such as folate and vitamin $B_{12}$ deficiency were ruled out by lab testing. Overall, the patient's normocytic anaemia was interpreted as a combination of anaemia of CKD exacerbated by blood loss in the urine.

The next step depends on if acute onset unilateral flank pain is present. If so, a workup for nephrolithiasis is indicated. The patient described an 


\begin{tabular}{llll}
\hline Table 1 & Complete blood count & & \\
\hline Component & Value & Reference range & Interpretation \\
\hline Haemoglobin & $67 \mathrm{~g} / \mathrm{L}$ & $135-175 \mathrm{~g} / \mathrm{L}$ & Low \\
Haematocrit & $20.3 \%$ & $42.0 \%-50.0 \%$ & Low \\
White blood cells & $5.7 \times 10^{9} / \mathrm{L}$ & $4-11 \times 10^{9} / \mathrm{L}$ & Normal \\
Platelets & $200 \times 10^{9} / \mathrm{L}$ & $150-450 \times 10^{9} / \mathrm{L}$ & Normal \\
\hline
\end{tabular}

insidious onset of mild right-sided flank pain, which is atypical of nephrolithiasis, nevertheless a non-contrast CT of the abdomen and pelvis was obtained in the ED to evaluate for stones. The imaging did not show any evidence of obstructions or hydronephrosis (figure 1).

The next diagnosis to workup is a urinary tract infection (UTI). The UA from the ED was positive for nitrates and trace leucocyte esterase, so a urine culture was sent to evaluate for infection. The culture came back positive for more than three organisms, which was considered to be the result of contamination. A second UA was negative for both nitrates and leucocyte esterase. Given the lack of clinical suspicion for UTI, repeat culture testing was withheld while more likely alternative diagnoses were pursued.

Laboratory data from admission also showed an elevated blood urea nitrogen (BUN) and creatinine (Cr), which suggested an acute kidney injury (AKI) on top of the patient's baseline stage III CKD (table 2). The BUN/Cr ratio of 7.9 and fractional excretion of sodium of $14 \%$ suggested that intrinsic or postrenal causes were more likely than prerenal causes. The most likely cause of this AKI was initially thought to be ischaemic acute tubular necrosis from blood loss.

Recommendations from a consultation with the nephrology service were to obtain an ultrasound of the kidney and bladder to evaluate for small ureteral strictures as well as serum protein electrophoresis (SPEP) and urine protein electrophoresis (UPEP) with immunofixation for the evaluation of multiple myeloma (MM). In addition, a QuantiFERON gold test was sent since urogenital tuberculosis (TB) may present with haematuria and is the third most common form of extrapulmonary TB. Moreover,

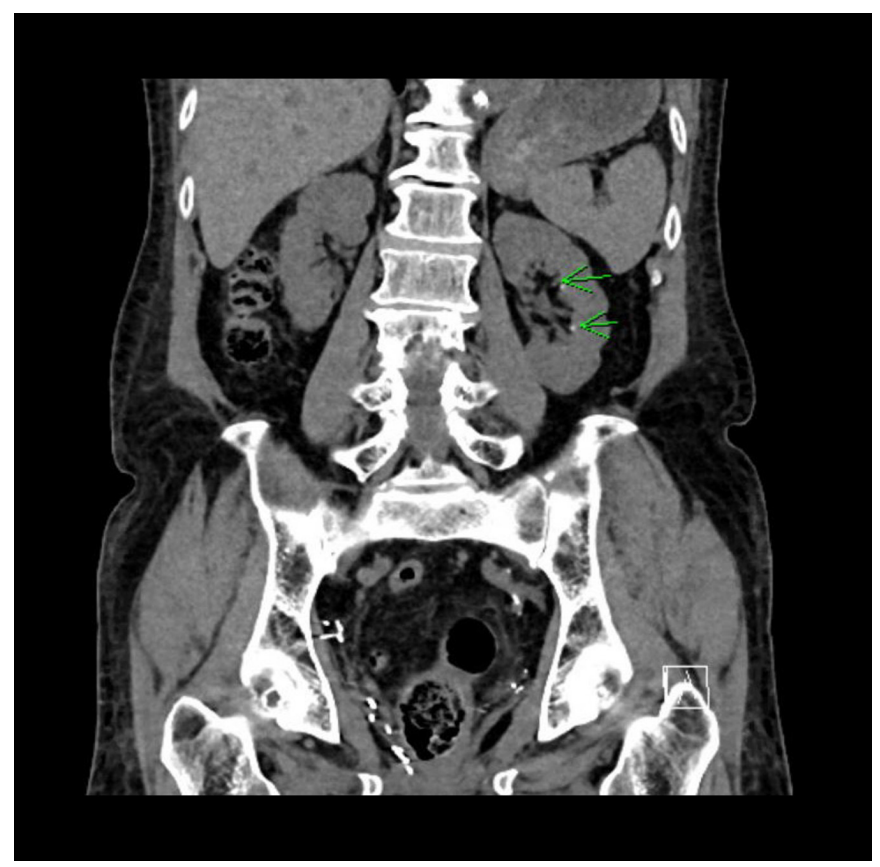

Figure 1 Non-contrast CT of the abdomen and pelvis showed $0.2 \mathrm{~cm}$ non-obstructing stones in the lower left pole of the kidney but no hydronephrosis.

\begin{tabular}{|c|c|c|c|}
\hline Component & Value & Reference range & Interpretation \\
\hline Blood urea nitrogen & $47 \mathrm{mg} / \mathrm{dL}$ & $2-25 \mathrm{mg} / \mathrm{dL}$ & High \\
\hline Serum $\mathrm{Cr}$ & $5.97 \mathrm{mg} / \mathrm{dL}$ & $0.60-1.3 \mathrm{mg} / \mathrm{dL}$ & High \\
\hline $\begin{array}{l}\text { Fractional excretion } \\
\text { of sodium }\end{array}$ & $14 \%$ & $\begin{array}{l}<1 \% \text { prerenal, } \\
>2 \% \text { intrinsic or } \\
\text { postrenal }\end{array}$ & $\begin{array}{l}\text { Intrinsic or } \\
\text { postrenal acute } \\
\text { kidney injury }\end{array}$ \\
\hline BUN/Cr ratio & 7.9 & $\begin{array}{l}>20 \text { prerenal, } \\
<20 \text { intrinsic or } \\
\text { postrenal }\end{array}$ & \\
\hline GFR & $9 \mathrm{~mL} / \mathrm{min}$ & $\geq 60 \mathrm{~mL} / \mathrm{min}$ & $\begin{array}{l}\text { End-stage renal } \\
\text { disease }\end{array}$ \\
\hline
\end{tabular}

Patient's baseline $\mathrm{Cr}$ was $1.3 \mathrm{mg} / \mathrm{dL}$, and baseline GFR was $42 \mathrm{~mL} / \mathrm{min}$.

BUN, blood urea nitrogen; $\mathrm{Cr}$, creatinine; GFR, glomerular filtration rate.

TB was certainly possible, given the patient's immigration from Southeast Asia.

The ultrasound of the kidney and bladder did not reveal any obstruction, and the lack of monoclonal gammopathy on SPEP and UPEP made MM unlikely. QuantiFERON gold testing for TB came back negative. By this point, the patient had been hospitalised for nearly a week with little improvement in BUN and $\mathrm{Cr}$, so the nephrology service recommended a renal biopsy. The main indication for performing a kidney biopsy in a patient with haematuria is the presence of urine albumin excretion above $30 \mathrm{mg} / \mathrm{d}$, which is suggestive of a glomerular aetiology. A measurement of the patient's 24-hour urine albumin level demonstrated excretion of $68.8 \mathrm{mg} / \mathrm{d}$. The patient consented to biopsy, and results returned on the same day.

Light microscopy showed that $40 \%$ of the glomeruli observed had cellular crescents, and some had necrotic lesions (figure 2). In addition, RBC casts were seen in many tubules. Electron microscopy of two glomeruli demonstrated slight focal thickening of the glomerular basement membrane and moderate expansion of the subendothelial space, but no wire-loop appearance. Finally, immunofluorescence of four glomeruli demonstrated high linear reactivity of anti-IgG, anti-kappa and anti-lambda, as well as moderate mesangiocapillary reactivity of anti-IgM, anti-C3 and antifibrinogen. Overall, these findings were most concerning for an anti-glomerular basement membrane (anti-GBM) glomerulonephritis. In a surprising twist, a previously unconsidered diagnosis quickly rose to the top of the differential.

Antibody titers were sent for anti-GBM, antinuclear antibody (ANA), anti-double-stranded DNA, perinuclear antineutrophil cytoplasmic antibodies/antimyeloperoxidase (p-ANCA/ anti-MPO) and cytoplasmic antineutrophil cytoplasmic antibodies/antiproteinase 3 (c-ANCA/anti-PR3), and complement C3 and C4 levels were checked. Slightly elevated levels of anti-MPO antibody at 21.4 AI and ANA at 1:80 were found. All other antibody titers were negative, and complement levels were normal. Data obtained later in his hospital course, after several sessions of plasmapheresis, were notable for normalisation of the level of anti-MPO antibody. In addition, nephrology recommended sending hepatitis $\mathrm{B}$ and $\mathrm{C}$ serologies (table 3 ). The patient's hepatitis serologies were consistent with latent hepatitis B infection without active viral replication and prior hepatitis $C$ infection without active viral replication.

At this time, the differential diagnosis included most causes of rapidly progressive glomerulonephritis (RPGN). Type I RPGN includes anti-GBM diseases such as the colloquially known Goodpasture's disease. Type II RPGN is characterised by high immune complex deposition and includes many causes. The aetiologies most relevant to this patient were lupus 


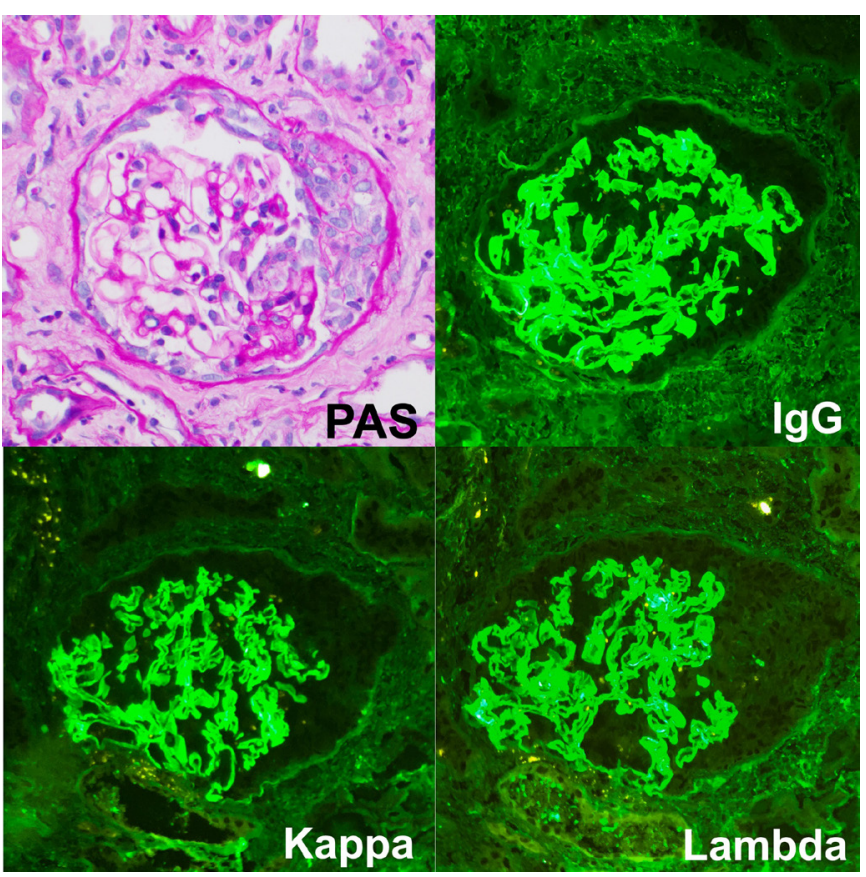

Figure 2 Kidney biopsy results: light microscopy of 20 glomeruli showed that eight of the glomeruli had cellular crescents, and two additional glomeruli had necrotic lesions. Red blood cell casts were seen in many tubules. Overall, about $20 \%$ of the renal parenchyma was effaced due to glomerulosclerosis, tubular atrophy, interstitial fibrosis and cellular infiltrates. Electron microscopy of two glomeruli demonstrated slight focal thickening of the glomerular basement membrane, occasional capillary loops and moderate expansion of the subendothelial space. Immunofluorescence of four glomeruli demonstrated high linear reactivity of anti-IgG, anti-kappa and antilambda, as well as moderate mesangiocapillary reactivity of anti-IgM, anti-C3 and anti-fibrinogen. PAS, periodic acid-Schiff.

nephritis, cryoglobulinemic membranoproliferative glomerulonephritis (MPGN) due to hepatitis C, and secondary membranous nephropathy $(\mathrm{MN})$ associated with solid tumour. Finally, type III RPGN is characterised by pauci-immune deposition and includes the small vessel vasculitides such as microscopic polyangiitis (MPA), granulomatosis with polyangiitis (formerly known as Wegner's granulomatosis) and eosinophilic granulomatosis with polyangiitis (EGPA, formerly known as ChurgStrauss syndrome).

Working through the differential, anti-GBM disease seemed most likely due to the linear IgG immunofluorescence and electron microscopy showing thickening of the glomerular basement membrane. However, anti-GBM disease is very rare with an incidence of 1 in 1.6 to 1.8 million and even more rarely presents

\begin{tabular}{|c|c|c|c|}
\hline Component & Value & Reference range & Interpretation \\
\hline Surface antigen & Negative & Negative & \multirow{5}{*}{$\begin{array}{l}\text { Carrier state with } \\
\text { no active viral } \\
\text { replication }\end{array}$} \\
\hline Surface antibody & $16 \mathrm{mlU} / \mathrm{mL}$ & $>9 \mathrm{mlU} / \mathrm{mL}$ & \\
\hline Core IgM antibody & Negative & Negative & \\
\hline Core $\lg G$ antibody & Positive & Negative & \\
\hline HBV PCR & $<20 \mathrm{IU} / \mathrm{mL}$ & $<20 \mathrm{IU} / \mathrm{mL}$ & \\
\hline Hepatitis C antibody & Positive & Negative & \multirow{2}{*}{$\begin{array}{l}\text { Prior infection } \\
\text { with no active viral } \\
\text { replication }\end{array}$} \\
\hline HCV PCR & $<15 \mathrm{IU} / \mathrm{mL}$ & $<15 \mathrm{IU} / \mathrm{mL}$ & \\
\hline
\end{tabular}

HBV, hepatitis B virus; HCV, hepatitis C virus.

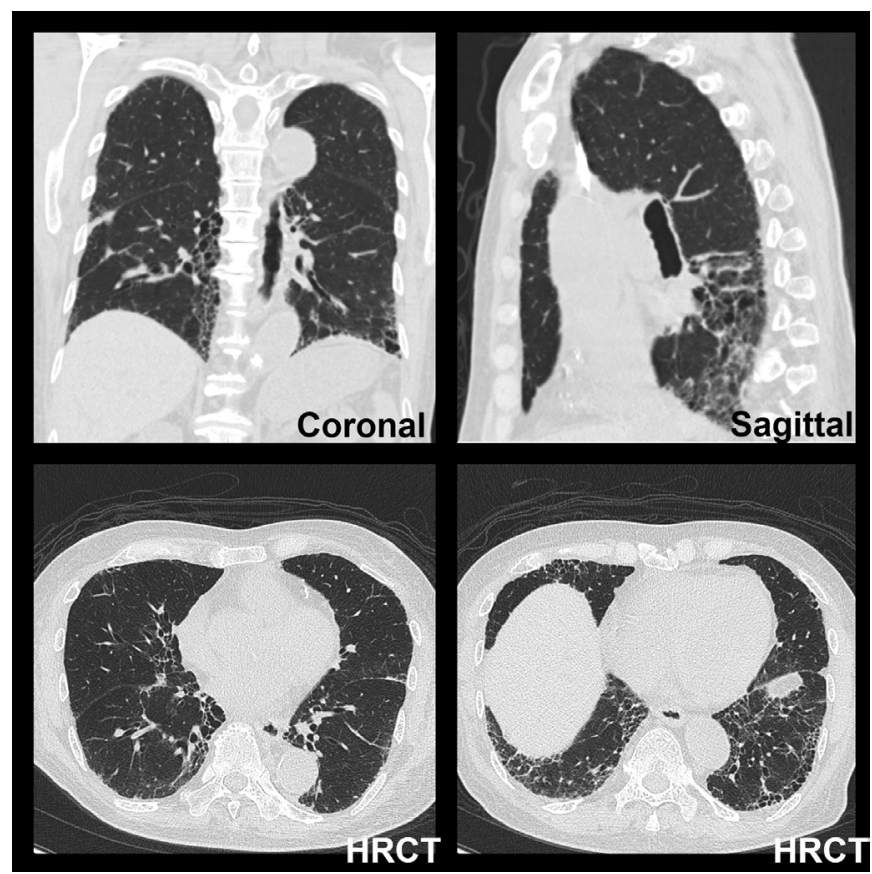

Figure 3 High-resolution CT (HRCT) of chest demonstrated basilar predominant peripheral fibrosis with honeycombing. Findings were compatible with usual interstitial pneumonia type pattern of fibrosis.

with negative anti-GBM antibody titers. Moreover, anti-GBM disease in which testing is negative for anti-GBM antibody and positive for anti-MPO antibody has only been documented in one other case report. Further testing with a Western blot for human Goodpasture antigen alpha-3 (IV) NC1 was considered. However, it was decided that the results would not influence the treatment plan, and so further testing was deferred.

Alternative diagnoses such as the type II RPGN's of lupus nephritis, cryoglobulinemic MPGN due to hepatitis $\mathrm{C}$ and secondary MN associated with solid tumour seemed less likely because immunofluorescence typically has a granular pattern as opposed to linear, and complement C3 and C4 levels are usually quite low. In addition, the typical wire-loop capillary appearance seen in lupus nephritis was not present. Additional antibody titers were sent for rheumatoid factor, and levels of cryoglobulin were checked to evaluate for cryoglobulinemic MPGN. All tests came back normal and further decreased the likelihood of these diagnoses.

The type III RPGN's seemed unlikely because the small vessel vasculitides usually present with a lack of immunofluorescence. Moreover, they often present with very high levels of anti-MPO or anti-PR3 antibody, usually much greater than the slightly elevated value of 21.4 AI for anti-MPO antibody seen in our patient. It is important to note that although anti-MPO antibody is most commonly associated with MPA and EGPA, it has also been known to be positive in some cases of anti-GBM disease.

In addition, a high-resolution CT (HRCT) of the chest was performed to further evaluate the pulmonary fibrosis detected on the CT of abdomen and pelvis from admission. The HRCT was consistent with usual interstitial pneumonia (UIP) fibrosis with no evidence of pulmonary vasculitis (figure 3 ). Pulmonary function tests confirmed a restrictive pattern of lung disease, and further evaluation was deferred to outpatient follow-up with pulmonology.

Overall, the final diagnosis was concluded to be a rare presentation of anti-GBM disease with negative anti-GBM antibody and positive anti-MPO antibody. The evidence for this diagnosis was strongly based on data from the renal biopsy including 
characteristic light and electron microscopy findings, as well as the linear immunofluorescence. Treatment for anti-GBM disease was started with special considerations for the patient's hepatitis B carrier state.

\section{TREATMENT}

On admission, the patient was anaemic with an $\mathrm{Hgb}$ of $67 \mathrm{~g} / \mathrm{L}$ and was transfused with 1 unit of packed RBCs. He was intermittently transfused during the hospital course to maintain a goal $\mathrm{Hgb}$ above $70 \mathrm{~g} / \mathrm{L}$.

Once anti-GBM disease was suspected based on the biopsy results, the patient was started on Solumedrol (methylprednisolone) $1 \mathrm{~g}$ intravenously for 3 days total and then given oral prednisone $60 \mathrm{mg}$ daily. A haemodialysis catheter was placed in the right internal jugular vein, and plasmapheresis was given every other day for a total of six sessions.

Therapeutic guidelines in anti-GBM disease are not well established; however, the current standard is to treat with intravenous methylprednisolone for 3 days, followed by an oral prednisone taper, in combination with oral Cytoxan/Neosar (cyclophosphamide) and plasmapheresis. Our approach was to begin with steroids and plasmapheresis and to withhold cyclophosphamide until we received results from the anti-GBM antibody titers.

It was during this initial treatment phase that the anti-GBM antibody titers came back negative, and the patient's carrier state for hepatitis B was discovered. The question of how long to continue plasmapheresis arose, since this therapy is usually used to decrease antibody titers, but our patient had negative levels to begin with. The decision was made to complete the six sessions of plasmapheresis given every other day, which was on the more conservative end of therapeutic guidelines, which suggest 2-3 weeks of therapy.

Following completion of plasmapheresis, the patient was started on Rituxan (rituximab) for immunosuppression and Baraclude (entecavir) for prophylaxis against hepatitis B virus (HBV) reactivation. Rituximab was chosen over cyclophosphamide, given the risks of haemorrhagic cystitis associated with the latter. Although current guidelines recommend cyclophosphamide, its efficacy in anti-GBM disease is unknown. Moreover, a few case reports have documented successful treatment of anti-GBM disease with rituximab. ${ }^{1}$ The rituximab was dosed at $1 \mathrm{~g}$ intravenously, given every 2 weeks.

Entecavir was chosen based on the results of a prospective study, which demonstrated a higher incidence of reactivation $(23 \%)$ in patients receiving rituximab who were HBsAg negative, anti-HBc positive and anti-HBs negative compared with patients who had almost the same serology profile but were anti-HBs positive. ${ }^{2}$ Since our patient fit the profile of the higher risk demographic, the decision was made to go forth with HBV prophylaxis. Of note, entecavir was chosen over Viread/Vemlidy (tenofovir disoproxil fumarate) because of the patient's low $\mathrm{Cr}$ clearance. Given the patient's Cr clearance of 11, the entecavir was renally dosed at $0.5 \mathrm{mg}$ given orally every 3 days. Finally, our patient was also started on renally dosed oral Bactrim (trimethoprim/sulfamethoxazole) tablet 160-800mg taken three times a week for prophylaxis against Pneumocystis jiroveci pneumonia.

\section{OUTCOME AND FOLLOW-UP}

The patient tolerated all treatments well and was deemed medically safe for discharge. Outpatient follow-up was arranged to continue rituximab treatment. In addition, follow-up visits were scheduled with nephrology to monitor renal function, pulmonology to further evaluate his fibrotic lung disease, radiation oncology and urology for prostate cancer and his primary care physician for management of his other chronic medical conditions.

Immunosuppressive therapy with rituximab and prednisone is usually continued for $2-3$ months until anti-GBM antibody titers return negative. In our patient, who presented with negative antibody titers, response to treatment is being assessed by recovery of renal function. HBV prophylaxis will be continued for at least 1 year after completion of rituximab since there is a lag in recovery of $\mathrm{B}$ cell function. He will also have monthly HBV PCR while on immunosuppression to monitor for reactivation.

At his follow-up visits with nephrology clinic, the patient's glomerular filtration rate (GFR) improved from $12 \mathrm{~mL} / \mathrm{min}$ at 1 month after discharge to $32 \mathrm{~mL} / \mathrm{min}$ at 2 months after discharge, compared with his prior baseline of $42 \mathrm{~mL} / \mathrm{min}$. The prognosis in anti-GBM disease is generally good with recovery of renal function in most patients who do not require dialysis on initial presentation. However, the prognosis in patients with atypical anti-GBM disease and history of stage III CKD is less clear.

\section{DISCUSSION}

There exists a broad spectrum of anti-GBM disease described in the literature. The classic example of Goodpasture's syndrome is positive for only anti-GBM antibody and has crescentic glomeruli on light microscopy. On one end of the spectrum is seronegative anti-GBM disease, which has recently been coined idiopathic linear immunoglobulin deposition (ILID). One study described about 20 such cases with the characteristic finding of no anti-GBM antibody and absent or focal crescents ( 8 out of 20 cases had $3 \%-40 \%$ of glomeruli with crescents). ${ }^{3}$ On the other end of the spectrum is double seropositive anti-GBM disease in which patients have anti-GBM antibody, ANCA and profuse crescentic glomeruli. Our patient-who had about $40 \%$ of glomeruli with crescents, negative anti-GBM antibody and positive anti-MPO antibody-represents a unique case that does not fit any of the usual patterns of disease.

Several case reports and review articles have demonstrated the relationship between anti-GBM antibody and ANCA. This phenomenon has been called double-positive anti-GBM disease as well as anti-GBM/ANCA cross-over disease. About one-third of patients with anti-GBM antibody are also positive for ANCA, usually p-ANCA/anti-MPO antibodies. ${ }^{4}$ Although the exact pathophysiologic nature of this relationship has not yet been elucidated, we do know that this relationship is not due to cross-reactivity. ${ }^{5}$ However, the occurrence of anti-GBM disease that is only positive for anti-MPO antibody is far rarer than the coexistence phenomenon.

Interestingly, patients aged older than 65 years are significantly more likely to present with double-positive serologies and renal impairment without haemoptysis compared with younger patients. In addition, although older patients were more likely to present with higher GFR, they were also more likely to develop end-stage renal disease (ESRD) than younger patients. ${ }^{6}$ Moreover, a multivariate Cox regression analysis showed that higher initial GFR was correlated with lower risk of ESRD and death within the older patient population. This demonstrates the importance of early detection in improving morbidity and mortality.

In addition, there is a known association between interstitial lung disease and the presence of anti-MPO antibody. This has been documented in several case reports and series. ${ }^{7}$ The most common type of interstitial lung disease found in these cases is UIP, which is the same finding identified in our patient. ${ }^{8}$ Another common characteristic to these cases is older age (around 70 years), which was also the age of our patient. ${ }^{9}$ Therefore, it is 
conceivable that our patient may have had two distinct disease processes. The first would be a pre-existing interstitial lung disease associated with anti-MPO antibody, and the second would be a glomerulonephritis of 1 month duration without an independent association with any antibodies. It is unknown how long anti-MPO antibody was present in our patient and thus whether the anti-MPO antibody was associated with his lung or kidney disease. In terms of prognosis, there is a high morality rate for patients who present with pulmonary fibrosis and the presence of anti-MPO antibody, which suggests the need for close monitoring of these patients.

Another phenomenon to be considered in our patient is radiation-induced glomerulonephritis. It is known that radiation may trigger an autoimmune response, and cases of anti-GBM glomerulonephritis following radiotherapy in patients with prostate cancer have been documented in the literature. ${ }^{10}$ However, anti-MPO antibody has not been found to be present in these patients. ${ }^{11}$ Interestingly, MPO is known to be present in prostatic epithelial cells. ${ }^{12}$ However, a clear relationship between prostate cancer, radiation therapy and the development of anti-MPO antibodies has not yet been demonstrated. Our patient presented with glomerulonephritis 2 weeks after completing radiotherapy for prostate cancer. Thus, it is quite possible that his glomerulonephritis was triggered by radiotherapy. Unlike other described cases, however, our patient was negative for anti-GBM antibodies and positive for anti-MPO antibody.

Only one other published abstract has documented a case of anti-GBM disease with negative anti-GBM antibody and positive anti-MPO antibody. The case described a patient who presented with haemoptysis but no haematuria and was found to have the same serologies and level of renal function as our patient. ${ }^{13}$ The patient described in that case was dialysis dependent on discharge. Interestingly, patients with double seropositive anti-GBM disease are more likely to recover from being dialysis dependent after treatment than patients only positive for anti-GBM antibody. ${ }^{14}$ However, double seropositive patients are also more likely to relapse than patients who only have anti-GBM antibody. The long-term outcomes of anti-GBM patients such as our own, who only have anti-MPO antibody, is as yet unknown. At his 2-month follow-up visit with nephrology clinic, our patient's GFR improved toward his baseline of stage III CKD, and his renal function will continue to be closely monitored.

Various mechanisms have been proposed to explain why anti-GBM disease may occur in the absence of anti-GBM antibody. The most relevant mechanism in our patient relates to antigen-antibody specificity. It is important to note that the Goodpasture antigen is the carboxyl terminal of the alpha-3 chain of type IV collagen ( $\alpha 3 \mathrm{NC} 1)$. In typical Goodpasture's disease, IgG1 and IgG3 antibodies form against $\alpha 3 \mathrm{NC} 1$. However, some cases have been described in which IgG4 antibodies are formed instead. ${ }^{3}$ This can cause false-negative antibody titers as detected by ELISA. ${ }^{15}$ Moreover, the IgG4 antibody poorly fixes complement, and thus, immunofluorescence of the GBM would be expected to lack complement C3 and C4. IgG4 was the predominant immunoglobulin in $67 \%$ of patients who presented with pauci-complement staining and positive stains for kappa and lamba light chain. This phenotype is called polytypic (as opposed to monotypic in which only kappa or lambda light chain stains positive) and is identical to the immunofluorescent staining pattern in our patient. This suggests that our patient may have been forming IgG4 antibodies to $\alpha 3 \mathrm{NC} 1$ instead of the usual IgG1 or IgG3. Overall, further studies are needed to determine the exact pathophysiology of ILID and other atypical anti-GBM diseases.
Learning points

- The colloquially known Goodpasture's disease actually exists on a spectrum from double seropositive disease to idiopathic linear immunoglobulin deposition, which does not have any antibodies.

- The mechanism of seronegative anti-glomerular basement membrane (GBM) disease is unclear, but one likely possibility is generation of $\operatorname{lgG} 4$ antibodies instead of $\mathrm{lgG1}$ or $\mathrm{lgG}$. Testing future patients for IgG subtypes would improve our understanding of this disease.

- The decision to use rituximab over cyclophosphamide depends on a risk versus benefit analysis for side effects such as haemorrhagic cystitis. In this case, the patient had moderate improvement of kidney function 2 months after initiating rituximab therapy.

- The diagnosis of glomerulonephritis with negative anti-GBM antibody and positive perinuclear anti-neutrophil cytoplasmic antibodies/antimyeloperoxidase antibody is exceedingly rare and has now been documented twice in the literature.

Contributors $\mathrm{AH}$ wrote and revised the manuscript and created tables, figures and images. JR took immunofluorescence photos and contributed to discussion section. FH contributed to discussion section.

Funding The authors have not declared a specific grant for this research from any funding agency in the public, commercial or not-for-profit sectors.

Competing interests None declared.

Patient consent for publication Obtained.

Provenance and peer review Not commissioned; externally peer reviewed.

Open access This is an open access article distributed in accordance with the Creative Commons Attribution Non Commercial (CC BY-NC 4.0) license, which permits others to distribute, remix, adapt, build upon this work non-commercially, and license their derivative works on different terms, provided the original work is properly cited, appropriate credit is given, any changes made indicated, and the use is non-commercial. See: http://creativecommons.org/licenses/by-nc/4.0/.

\section{REFERENCES}

1 Touzot M, Poisson J, Faguer $\mathrm{S}$, et al. Rituximab in anti-GBM disease: A retrospective study of 8 patients. J Autoimmun 2015;60:74-9.

2 Hsu C, Tsou HH, Lin SJ, et al. Chemotherapy-induced hepatitis B reactivation in lymphoma patients with resolved HBV infection: a prospective study. Hepatology 2014;59:2092-100.

3 Nasr SH, Collins AB, Alexander MP, et al. The clinicopathologic characteristics and outcome of atypical anti-glomerular basement membrane nephritis. Kidney Int 2016;89:897-908.

4 Levy JB, Hammad T, Coulthart A, et al. Clinical features and outcome of patients with both ANCA and anti-GBM antibodies. Kidney Int 2004;66:1535-40.

5 Yang R, Hellmark T, Zhao J, et al. Antigen and epitope specificity of anti-glomerular basement membrane antibodies in patients with goodpasture disease with or without anti-neutrophil cytoplasmic antibodies. J Am Soc Nephrol 2007;18:1338-43.

6 Cui Z, Zhao J, Jia XY, et al. Clinical features and outcomes of anti-glomerular basement membrane disease in older patients. Am J Kidney Dis 2011;57:575-82.

7 Kishore N, Gupta N, Dhar A, et al. Interstitial lung disease with usual interstitial pneumonia pattern preceding the presentation of ANCA-associated vasculitis by 4 years: coincidence or correlation? Breathe 2018;14:e105-10.

8 Katsumata Y, Kawaguchi Y, Yamanaka H. Interstitial Lung Disease with ANCAassociated Vasculitis. Clin Med Insights Circ Respir Pulm Med 2015;9(Suppl 1):CCRPM.S23314-56.

9 Yamada H. ANCA: associated lung fibrosis. Semin Respir Crit Care Med 2011;32:322-7.

10 Nasrullah A, Fatima Z, Javed A, et al. A Case of Anti-glomerular Basement Disease Without Pulmonary Involvement. Cureus 2019;11:4130.

11 Sugiyama M, Yamada Y, Nozaki Y, et al. Anti-glomerular basement membrane glomerulonephritis after radiotherapy for early prostate cancer. Clin Kidney J 2014;7:90-1.

12 Roumeguère T, Delree $\mathrm{P}$, Van Antwerpen $\mathrm{P}$, et al. Intriguing location of myeloperoxidase in the prostate: a preliminary immunohistochemical study. Prostate 2012;72:507-13.

13 Azam M0, Thimmisetty R, Adekola 0, et al. Antibody negative Goodpasture syndrome-a rare entity. Am J Kidney Dis 2016;67:26. 
14 McAdoo SP, Tanna A, Hrušková Z, et al. Patients double-seropositive for ANCA and anti-GBM antibodies have varied renal survival, frequency of relapse, and outcomes compared to single-seropositive patients. Kidney Int 2017;92:693-702.
15 Ohlsson S, Herlitz H, Lundberg S, et al. Circulating anti-glomerular basement membrane antibodies with predominance of subclass lgG4 and false-negative immunoassay test results in anti-glomerular basement membrane disease. Am J Kidney Dis 2014;63:289-93.

Copyright 2019 BMJ Publishing Group. All rights reserved. For permission to reuse any of this content visit

https://www.bmj.com/company/products-services/rights-and-licensing/permissions/

BMJ Case Report Fellows may re-use this article for personal use and teaching without any further permission.

Become a Fellow of BMJ Case Reports today and you can:

- Submit as many cases as you like

- Enjoy fast sympathetic peer review and rapid publication of accepted articles

Access all the published articles

Re-use any of the published material for personal use and teaching without further permission

\section{Customer Service}

If you have any further queries about your subscription, please contact our customer services team on +44 (0) 2071111105 or via email at support@bmj.com.

Visit casereports.bmj.com for more articles like this and to become a Fellow 